\title{
Refraction effects on x-ray and ultraviolet interferometric probing of laser-produced plasmas
}

\author{
Raymond F. Smith, James Dunn, Joseph Nilsen, and James R. Hunter
}

Lawrence Livermore National Laboratory, Livermore, California 94551

Vyacheslev N. Shlyaptsev

Department of Applied Science, University of California Davis-Livermore, Livermore, California 94551

Jorge J. Rocca and Jorge Filevich

Department of Electrical and Computer Engineering, Colorado State University, Fort Collins, Colorado 80523

\author{
Mario C. Marconi
}

Department of Electrical and Computer Engineering, Colorado State University, Fort Collins, Colorado 80523, and Department of Physics, University of Buenos Aires, Ciudad Universitaria-C1428EHA, Argentina

Received April 5, 2002; revised manuscript received September 9, 2002

\begin{abstract}
We present a study detailing the effects of refraction on the analysis and interpretation of line-of-site optical probe characterization techniques within laser-produced plasmas. Results using x-ray laser backlit grid deflectometry and ray-tracing simulations illustrate the extent to which refraction can be a limiting factor in diagnosing high-density, short-scale-length plasmas. Analysis is applied to a recent experiment in which soft$\mathrm{x}$-ray interferometry was used to measure the electron density within a fast-evolving Al plasma. Comparisons are drawn between extreme ultraviolet and ultraviolet probe wavelengths. (c) 2003 Optical Society of America OCIS codes: $120.3180,120.5710$.
\end{abstract}

\section{INTRODUCTION}

Optical probing of laser-produced plasmas has been widely employed to yield direct measurements of a number of plasma parameters, such as electron density, ${ }^{1}$ electron temperature, ${ }^{2}$ and expansion velocity of the critical surface. $^{3}$ Valuable insights have been obtained into laser-plasma interactions such as beam channeling, filamentation, ${ }^{4}$ and profile steepening resulting from pondermotive forces. ${ }^{5}$ These powerful diagnostic techniques provide the empirical data necessary for benchmarking and validating hydrodynamic plasma codes, which in turn further our understanding of the underlying physical processes involved in the experiment.

An important consideration in the analysis of all plasma probing experiments is the extent to which probe beam is deflected, by refraction, during propagation within the plasma medium. Laser-produced plasmas typically have large density gradients over spatially small scale lengths. Under such conditions refraction can have a significant effect on steering the probe beam away from regions of high density. Within the frame under study, deflection may result in the probing of very different plasma conditions than on the original path. Knowledge of the extent and effects of refraction is, therefore, an important consideration when the accuracy of the analyzed data is to be determined. In this paper an experimental and theoretical analysis is presented that quantifies how refraction affects a $14.68-\mathrm{nm}$ Ni-like Pd x-ray laser used to probe an $\mathrm{Al}$ plasma with a diffraction-grating interferometer technique. ${ }^{1,6}$ Many other plasma interferometry experiments have used UV wavelength probes, ${ }^{4,5}$ and so in our analysis comparisons are drawn on the effectiveness of using probe wavelengths at $265 \mathrm{~nm}$ and at $14.68 \mathrm{~nm}$.

\section{EXPERIMENTAL SETUP}

A skewed Mach-Zehnder interferometer, with diffraction gratings as beam splitters, was used to diagnose the twodimensional electron density evolution of an $\mathrm{Al}$ plasma. ${ }^{1,6}$ The probe beam used was the $14.68 \mathrm{~nm}$ Ni-like Pd x-ray laser output generated with the standard experimental arrangement on the COMET laser facility at the Lawrence Livermore National Laboratory. ${ }^{7}$ Recent experiments have characterized this scheme as yielding tens of millijoules of $\mathrm{x}$-ray laser photon energy within a pulse width of a few picoseconds. ${ }^{7,8}$ The picosecond pulse duration mitigates effects of plasma motion blurring and allows effective snapshots of the electron density profile to be taken within $10 \mu \mathrm{m}$ of the target surface. ${ }^{1}$

The experimental arrangement is described in Fig. 1. Upon entering the diffraction grating interferometer, the 


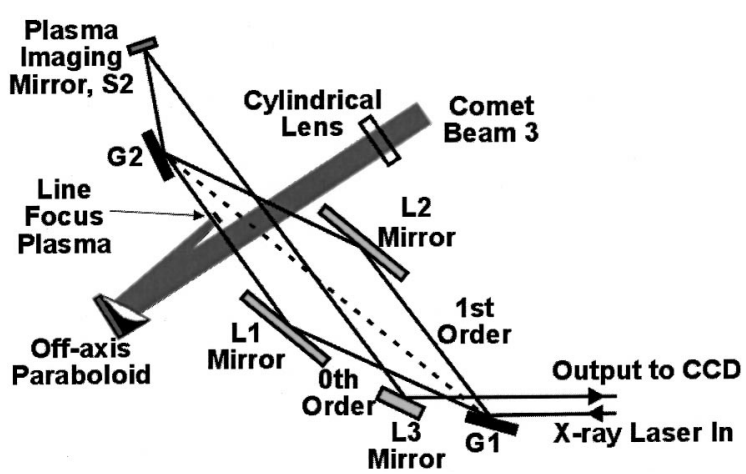

Fig. 1. Experimental setup for $\mathrm{x}$-ray laser diffraction grating interferometry on the COMET laser.

$\mathrm{x}$-ray laser pulse is incident onto a Au-coated $900-$ line/mm grating (G1). The blaze angle on the grating is compensated to equalize the intensity within the zeroth order and -1 st order arms (25\% each). The two diverging arms are reflected at a $2.6^{\circ}$ grazing incidence from $0.3-\mathrm{m}$ long Au-coated mirrors (L1 and L2) onto the center of a second grating, G2. After G2 the first order of the -1 st input arm $\left(-1_{1}\right)$ and the zeroth order of the zeroth-order input arm $\left(0_{0}\right)$ are copropagating, and if the temporal and spatial coherence conditions are met interference fringes will be generated. The x-ray laser is then imaged by a 0.25-m-focal-length spherical mirror (S2) at $\sim 5^{\circ}$ off normal incidence and routed to a CCD detector at grazing incidence off a long Au-coated mirror, L3.

Plasma probing is achieved by generating a laserproduced plasma along the trajectory of the zeroth-order arm. For this we introduced a third beam from the COMET laser, which could provide up to $3 \mathrm{~J}$ of energy in a 600-ps pulse at $1054 \mathrm{~nm}$. A 2-m-focal-length cylindrical lens was inserted into the $40-\mathrm{mm}$-diameter beam path before incidence onto a $0.3-\mathrm{m}$-focal-length off-axis paraboloid. This generated a $6 \times 0.02-\mathrm{mm}^{2}$ line focus on the target plane. This geometry facilitated longitudinal probing of the line focus plasma by the x-ray laser, thereby minimizing uncertainties in the interpretation of the interferograms arising from plasma gradients along the probe path.

To position the output of the target plane in the focal plane of $\mathrm{S} 2$, a $12.7-\mu \mathrm{m}$ spacing mesh was placed perpendicular to the target surface and was backlit by the x-ray laser beam. The target was then translated until the mesh, and hence the output plane, was in focus. This enabled the imaging system to be characterized as having a magnification of $9.94 \times$ with a pixel-limited resolution of $2.55 \mu \mathrm{m}$ at the target plane. A 1-mm pinhole placed between G2 and S2 (see Fig. 4 below) minimized the collection cone of the imaging system to reduce the signal associated with the plasma x-ray self-emission on the recorded interferograms. The acceptance angle imposed by the pinhole ensured that rays associated with the probe beam refracted more than 3 mrad would not be detected.

\section{RESULTS AND REFRACTION ANALYSIS}

In interferometry fringe shifts associated with density gradients make two-dimensional mapping of electron density profiles possible. It is, however, the presence of the electron density gradients that causes refraction and that can limit the accuracy of the electron density measurements. Rays associated with the probe beam that initially propagate parallel to the target surface will tend to be deflected toward regions of lower electron density. Assuming a static plasma profile over the interval of probing, the angle of refraction may be approximated from the paraxial ray equation ${ }^{9}$ as

$$
\theta \approx L\left(\frac{n_{e}(z)}{2 n_{\mathrm{crit}} n_{L}}\right),
$$

where $L$ is the length of the plasma; $z$ is the distance from the target; $n_{\text {crit }}\left(\mathrm{cm}^{-3}\right)$ is the critical density, given as $n_{\text {crit }}=1.1 \times 10^{21} \lambda^{-2}$, with the probe wavelength $\lambda$ in micrometers; and $n_{L}$ is the density scale length, given as

$$
n_{e}(x)=n_{e o} \exp \left(-\frac{z}{n_{L}}\right) \text {. }
$$

The critical density for the Ni-like Pd x-ray laser at 14.68 $\mathrm{nm}$ is $5.2 \times 10^{24} \mathrm{~cm}^{-3}$ and for a $265-\mathrm{nm}$ UV probe beam is $1.8 \times 10^{22} \mathrm{~cm}^{-3}$. This represents the ultimate limit on the electron density that these wavelengths can probe. It is clear from relation (1) that refractive effects are minimized for shorter target lengths and for shorterwavelength probes.

For a plasma with an electron density $n_{e}$, the index of refraction $n_{\text {ref }}$ of the plasma is related to $n_{\text {crit }}$ by

$$
n_{\text {ref }}=\left(1-n_{e} / n_{\text {crit }}\right)^{1 / 2} .
$$

The index of refraction of the plasma in one arm of an interferometer introduces a fringe shift, $N_{\text {fringe }}$, given by ${ }^{10}$

$$
N_{\text {fringe }}=\frac{\delta \psi}{2 \pi}=\frac{1}{\lambda} \int_{0}^{L}\left(1-n_{\text {ref }}\right) \mathrm{d} l \approx \frac{n_{e}}{2 n_{\text {crit }}} \frac{L}{\lambda},
$$

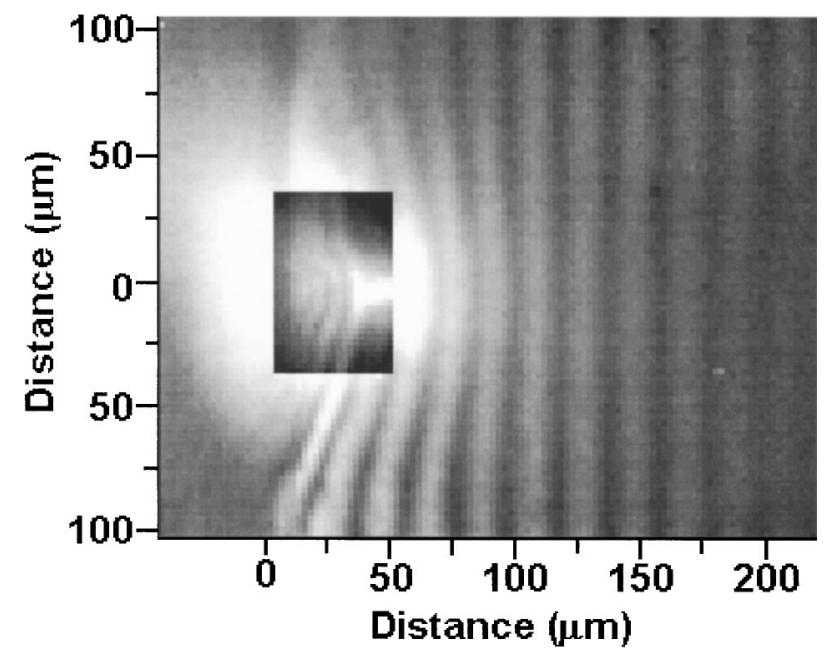

Fig. 2. A 5-mm-long Al plasma is probed at $700 \mathrm{ps}$ after the peak of the 600-ps (FWHM) plasma forming beam by the 14.68-nm Ni-like Pd x-ray laser. The plasma was generated with a $6 \times 0.04 \mathrm{~mm}^{2}$ line focus with an intensity of 4 $\times 10^{11} \mathrm{~W} / \mathrm{cm}^{2}$. Each fringe shift is equivalent to an electron density of $2.95 \times 10^{19} \mathrm{~cm}^{-3}$. Different gray scales are applied to the image for clarity. 


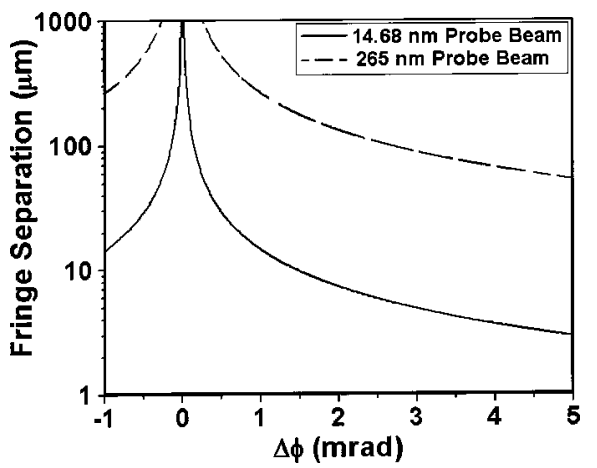

Fig. 3. Fringe separation for two different probe wavelengths as a function of the relative angle, $\Delta \phi$, of the combing phase fronts associated with the two arms of the interferometer.

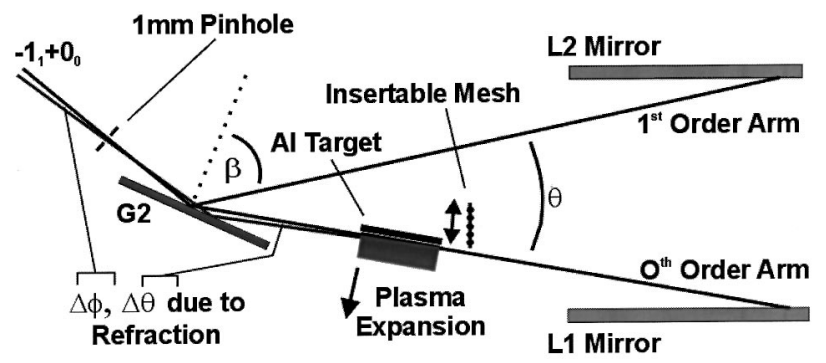

Fig. 4. Refraction away from the target surface has a direct relation to the local relative angle $(\Delta \phi)$ of the combining phase fronts of the two arms after G2. The 1-mm pinhole after G2 prevents rays from the probe beam refracted greater than $3 \mathrm{mrad}$ from being accepted by the imaging system. The insertable 12.7- $\mu \mathrm{m}$ spacing mesh allows grid deflectometry measurements of the refraction to be measured (see Fig. 5).

where $\delta \psi$ is the relative phase change and $L$ is the plasma length. Therefore, when the fringe shifts at the output plane of the plasma are accurately measured, a twodimensional electron density map of the plasma expansion profile can be constructed. A typical interferogram is shown in Fig 2.

The fringe frequency in the absence of a plasma, $K$ (radians per meter), gives a measure of the local relative angle, $\Delta \phi$, of the recombined phase fronts in the two interferometer arms through the relation ${ }^{11}$

$$
K=\frac{2 \pi \sin \Delta \phi}{\lambda}
$$

The relationship between fringe separation and $\Delta \phi$ is shown in Fig. 3 for two different probe wavelengths. A small fringe separation is desirable to yield detailed spatial information but in practice this is limited by the achievable resolution of the imaging system. In Fig. 4 a detailed schematic diagram of the recombining section of the interferometer is shown. The first-order and zerothorder arms are, respectively, reflected off the L2 and L1 $\mathrm{Au}$ mirrors onto G2. The output angle of the zerothorder arm from G2 is equal to its input angle. The output angle of the first-order arm, $\phi$, is determined by the grating equation,

$$
\phi=\sin ^{-1}\left(\frac{m \lambda}{d}-\sin \beta\right),
$$

where $\beta$ is the input angle, $m$ is the diffraction order, and $d$ is the lines per millimeter on the grating. Therefore, for a given relative angle $\theta$ between the two orders upon incidence onto G2, the relative combining angle between the two orders, $\Delta \phi$, is set. This sets the fringe separation according to the relationship in Eq. (5) and Fig. 3. Experimentally this fringe separation was accurately set by the horizontal rotation of L2 with good agreement with the predicted values. In our experimental setup the unperturbed fringe separation was fixed at $20 \mu \mathrm{m}$. To ensure that fringe shifts occurred away from the target surface in the presence of the plasma, it was necessary to reduce the value of $\theta$ from the value that gives $\Delta \phi=0$ (null position). Increasing $\theta$ from the null position causes fringe shifts to go toward the target surface in the presence of a plasma.

Introducing refraction into the zeroth-order arm locally modifies the input angle onto G2, which translates into a new local angular offset, $\Delta \phi$, between the two combining arms. The net result is to locally reduce $\theta$, which ultimately has the effect of reducing the fringe separation according to the relation given in Eq. (5). It can be seen, for example, in Fig. 3 that if rays were refracted uniformly by $1 \mathrm{mrad}$ the fringe separation would be reduced from 20 $\mu \mathrm{m}$ to $\sim 10 \mu \mathrm{m}$. The extent of the fringe separation change is dependent on the initial fringe spacing. In addition, owing to the relatively larger $\Delta \phi$ required for a 265-nm probe to achieve an initial fringe separation of, say, $20 \mu \mathrm{m}$ (Fig. 3), it would be expected that UV wavelengths would be more insensitive to this effect.

Refraction may be manifested on the interferograms in other ways. Beams that are refracted will have a different path length than the nonrefracted rays. Hence a larger phase differential will exist when these rays are combined with the first-order arm. This is further complicated by different electron density regions sampled along the new trajectory. Beams refracted completely away from a given region out of the collection angle of the imaging system would result in the loss of fringes with only the signal from the first-order arm remaining. As refraction is most severe closest to the target surface, this ultimately places a limit on how close to that surface the probe beam can diagnose.

For short-scale-length plasma studies refraction may add significant uncertainty to results that attribute fringe shifts solely to phase differentials between the two orders due to the refractive index's dependency on electron density. Therefore in regions where refraction is problematic misestimations of electron density can occur. It is important, therefore, to quantify the extent of refraction within our setup.

\section{GRID DEFLECTOMETRY}

To experimentally study the effects of refraction, we placed a $12.7-\mu \mathrm{m}$ spacing $\mathrm{Ni}$ mesh in front of the plasma and backlit it with the zero-order arm (Fig. 4). The x-ray laser (with the shadow of the mesh) traversed the plasma with refraction tending to deflect the image of the mesh away from the target. The image shown in Fig. 5 represents the backlit mesh traversing longitudinally through a 5-mm-long $\mathrm{Al}$ plasma under the same irradiance condi- 


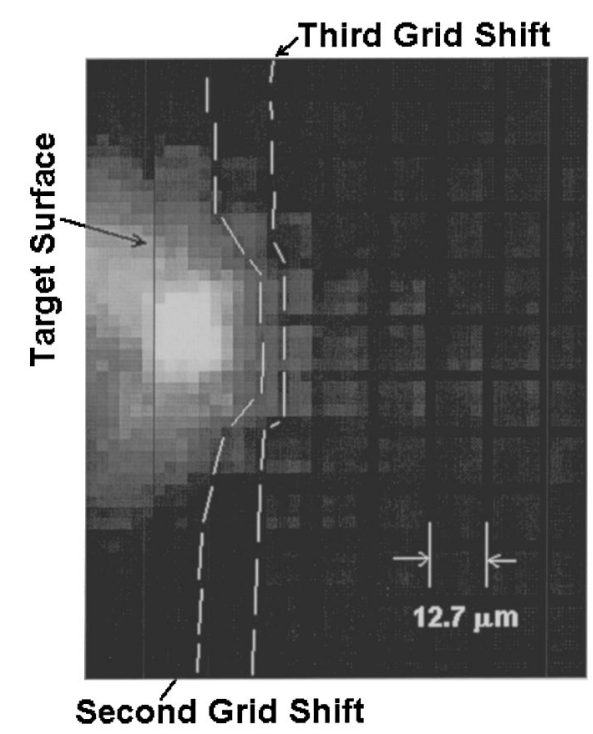

Fig. 5. A 12.7- $\mu \mathrm{m}$ spacing mesh is placed in front of the target (as shown in Fig. 4) and backlit by the probe beam to allow grid deflectometry measurements of the refraction to be made. Different gray scales have been applied for reproducibility.

tions of Fig. 2. The x-ray laser probed $+0.5 \mathrm{~ns}$ after the peak of the 600-ps plasma-forming pulse. In the resultant image, the grid (third grid) initially $24.8 \mu \mathrm{m}$ away from the target surface was displaced by $4.5 \mu \mathrm{m}$. This corresponds to a deflection angle of $0.89 \mathrm{mrad}$. The grid, which was initially at $13.3 \mu \mathrm{m}$, was deflected by $11 \mu \mathrm{m}$. This equates to a deflection angle of $2.2 \mathrm{mrad}$. The shadow of the first grid from the target surface was masked by the x-ray self-emission from the plasma. Because of the scaling with length, for a 1-mm target, the $\mathrm{x}$-ray laser rays propagating initially $13.3 \mu \mathrm{m}$ away from the target would undergo a $\sim 0.44$-mrad deflection, with larger refraction angles for rays closer to the target. This was the preferred target length for the experiment. In our setup the angles of refraction map directly onto $\Delta \phi$ and alter the local fringe separation accordingly (Fig. 3). Owing to the $\sim \lambda^{2}$ dependence of refraction, $a \sim 0.44 \mathrm{mrad}$ deflection at $14.68 \mathrm{~nm}$ would be equivalent to $\sim 125 \mathrm{mrad}$ if a $265 \mathrm{~nm}$ probe wavelength were used.

\section{RAY TRACING}

Figure 6 shows the on-axis electron density profile measured from the $\mathrm{x}$-ray laser longitudinal probing of a 1-mm-long Al plasma. Irradiance was achieved with a $6 \times 0.02 \mathrm{~mm}^{2}$ line focus with an on-target intensity of $1.25 \times 10^{12} \mathrm{~W} / \mathrm{cm}^{2}$. The probe time is $500 \mathrm{ps}$ after the peak of the 600-ps plasma-forming pulse. The calculated electron density from the RADEX ${ }^{12}$ plasma physics code and the predicted modification of this electron density profile when observed by interferometry, with refraction effects considered, are also plotted as a function of the initial distance from the target surface. We take refraction into account by ray tracing through the millimeter long plasma while also considering the effect of the changes in the relative angle of the phase front. Results are shown for the 14.68-nm probe wavelength, assuming an initial fringe separation of $20 \mu \mathrm{m}$ and neglecting the effect of rays with refraction angles greater than $3 \mathrm{mrad}$ (to agree with the experimental conditions). The RADEX onedimensional simulations were run under the same intensity conditions as the experiment but with an $80-\mu \mathrm{m}$ line focus to compensate for lateral plasma expansion larger than the initial line focus width. ${ }^{1}$ This gave close agreement with the measured on-axis density profile as shown in Fig. 6. The calculated electron density continues to increase by several orders of magnitude within a few micrometers of the target surface. This results in higher refraction, which prohibits extensive sampling by the x-ray laser probe beam over the millimeter plasma length. The extent of the refraction for the two probe wavelengths is calculated by a ray-tracing postprocessor applied to the calculated RADEX electron density profile. The dominant effect that causes disagreement between the extreme ultraviolet (XUV) diagnosis and the predicted electron density is the local modification by refraction of the angular offset, $\Delta \phi$, between the two combining arms of the interferometer. Owing to the plasma geometry and the spatial resolution of the imaging system, the x-ray laser probe is predicted to diagnose the plasma to within $\sim 13 \mu \mathrm{m}$ of the target surface. The effect of refraction contributes to a $<20 \%$ overestimation in electron density for $\sim 3 \times 10^{20} \mathrm{~cm}^{-3}$ at $z=13 \mu \mathrm{m}$.

The ray-traced trajectories of the UV and XUV probe wavelengths through the plasma are shown in Fig. 7 as a function of distance from the target surface. Refraction effects are predicted to preclude probing of the $265 \mathrm{~nm}$ beam within $\sim 75 \mu \mathrm{m}$ from the target surface for a 1-mm target length. Even above this limit, interpretation of the interferograms would be complicated by a number of factors. At the output plane between 75-80 $\mu \mathrm{m}$, for example, the integrated signal is a contribution of rays that initially propagated $30-60 \mu \mathrm{m}$ from the target surface. Each of these rays has a different $n_{e} L$ product because of their differing trajectories through the plasma. The overall trend, therefore, would be for an averaged sampled density exceeding that of a ray that was unaffected by refraction and that maintained a distance of 75

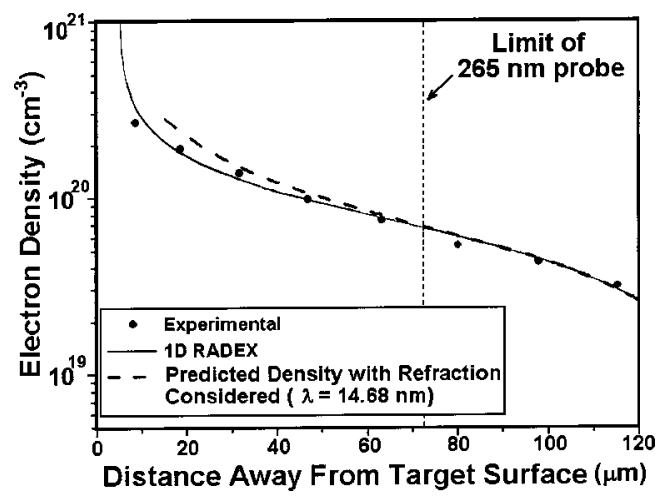

Fig. 6. Measured electron density (circles), RADEX onedimensional calculated density profile (solid curve) and the predicted modification of this profile (dashed curve), when viewed with an interferometer with refraction effects considered (1-mm target length), shown as a function of distance away from the target surface $(\lambda=14.68 \mathrm{~nm})$. The $265-\mathrm{nm}$ probe beam is limited by refraction to $>75 \mu \mathrm{m}$ from the target surface with the predicted limit of the 14.68-nm probe wavelength, shown by the termination of the dashed line, at $\sim 13 \mu \mathrm{m}$. 


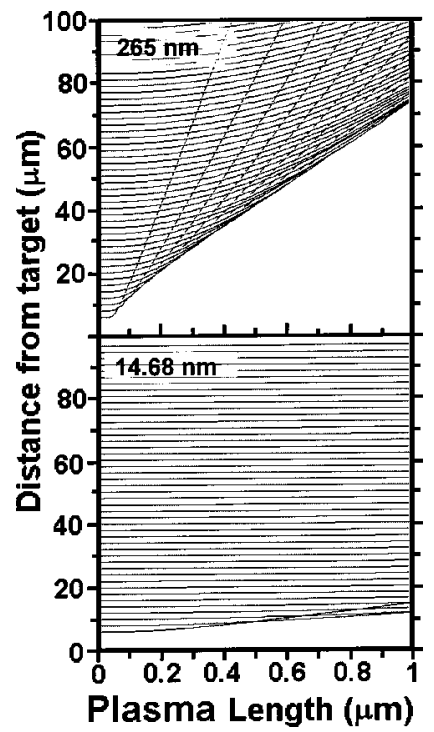

Fig. 7. Ray tracing of the probe beam through the calculated RADEX density profile shown in Fig. 6. Two probe wavelengths, 14.68 and $265 \mathrm{~nm}$, are considered for target lengths as great as $1 \mathrm{~mm}$.

$\mu \mathrm{m}$ from the target surface during propagation. In addition, all of these rays have different angles associated with their phase fronts. Both of these effects would combine to have a significant contribution in determining the fringe structure in the interferogram. The quality of the fringes would further be compromised by the extensive overlap, at the output plane, of rays that were initially adjacent. In the case where spatial overlap is expected to be significant, grid deflectometry techniques would not be useful to experimentally diagnose the extent of refraction on the $265 \mathrm{~nm}$ beam. In this region the density profile would require to be known a priori to permit us to understand the effect of refraction on the final interferogram.

For the density profile under study large deflection angles preclude the UV probe from diagnosing large plasmas $(>50 \mu \mathrm{m})$. For this reason the use of UV probes in the diagnostics of high-density plasmas is mostly limited to spot-focus plasmas of small dimensions. However, in this case Abel inversion techniques, which depend on the absolute symmetry of the plasma, must be used to extract the density profile. In addition, there is strong wavelength dependence on free-free absorption $\left[\propto \lambda^{3}[1\right.$ $-\exp (-h c / \lambda k T)]]$ which is expected to attenuate the UV probe beam in large plasmas.

Both simulations and grid deflectometry measurements provide insight into the plasma gradients and effects that are due to refraction on the interferograms in a rigorous manner. Using these techniques to quantify the extent of refraction allows the resultant effects on the measured density profiles to be removed in a selfconsistent manner. This is possible when refraction does not strongly overlap initially adjacent beam trajectories at the target output plane.

\section{CONCLUSIONS}

Refraction effects within dense, short-scale-length plasmas can impose significant deflection on optical probe beams during propagation. In interferometric studies of laser produced plasmas extensive refraction can lead to errors in the electron density measurements. Grid deflectometry and ray tracing can accurately diagnose the extent of refraction, which may be then deconvolved from the measured data. Refraction is highly dependent on the wavelength of the probe beam. We have shown that for probing dense short-scale-length plasmas greater than $\sim 50 \mu \mathrm{m}$ in longitudinal dimension it is necessary to probe in the soft-x-ray range. X-ray lasers are well suited to probe large millimeter scale plasmas found in hohlraums. ${ }^{13}$

\section{ACKNOWLEDGMENTS}

The support of $\mathrm{Al}$ Osterheld and Andy Hazi is greatly appreciated. The authors are pleased to acknowledge the technical contributions from Carl Bruns and Al Ellis. This work was performed under the auspices of the U.S. Department of Energy by the University of California Lawrence Livermore National Laboratory, through the Institute for Laser Science and Applications, under contract W-7405-Eng-48 and U.S. Department of Energy grant DE-FG03-98DP00208. The development of the interferometer's gratings was supported with a grant from the State of Colorado Photonics and Optoelectronics program.

R. F. Smith's e-mail address is smith248@llnl.gov.

\section{REFERENCES}

1. R. F. Smith, J. Dunn, J. Nilsen, V. N. Shlyaptsev, S. Moon, J. Filevich, J. J. Rocca, M. C. Marconi, J. R. Hunter, and T. W. Barbee, Jr., "Picosecond x-ray laser interferometry of dense plasmas," Phys. Rev. Lett. 89, 065004-1 (2002).

2. L. Aschke, S. Depierreux, K. G. Estabrook, K. B. Fournier, J. Fuchs, S. Glenzer, R. W. Lee, W. Rozmus, R. S. Thoe, and P. E. Young, "Towards an experimental benchmark for aluminum x-ray spectra," J. Quant. Spectrosc. Radiat. Transf. 65, 23-30 (2000).

3. J. P. Geindre, P. Audebert, A. Rousse, F. Fallies, J. C. Gauthier, A. Mysyrowicz, A. Dos Santos, G. Hamoniaux, and A. Antonetti, "Frequency-domain interferometer for measuring the phase and amplitude of a femtosecond pulse probing a laser-produced plasma," Opt. Lett. 19, 1997-1999 (1994).

4. P. E. Young, C. H. Still, D. E. Hinkel, W. L. Kruer, E. A. Williams, R. L. Berger, and K. G. Estabrook, "Observations of laser-beam bending due to transverse plasma flow," Phys. Rev. Lett. 81, 1425-1428 (1998).

5. D. A. Attwood, D. W. Sweeney, J. M. Auerbach, and P. H. Y. Lee, "Interferometric confirmation of radiation-pressure effects in laser-plasma interactions," Phys. Rev. Lett. 40, 184-187 (1978).

6. J. Filevich, K. Kanizay, M. C. Marconi, J. L. A. Chilla, and J. J. Rocca, "Dense plasma diagnostics with an amplitudedivision soft-x-ray laser interferometer based on diffraction gratings," Opt. Lett. 25, 356-358 (2000).

7. J. Dunn, Y. Li, A. L. Osterheld, J. Nilsen, J. R. Hunter, and V. N. Shlyaptsev, "Gain saturation regime for laser-driven tabletop, transient Ni-like ion X-ray lasers," Phys. Rev. Lett. 84, 4834-4837 (2000).

8. A. Klisnick, J. Kuba, D. Ros, R. Smith, G. Jamelot, C. Chenais-Popovics, R. Keenan, S. Topping, C. L. S. Lewis, F. Strati, G. J. Tallents, D. Neely, R. Clarke, J. Collier, A. G. 
MacPhee, F. Bortolotto, P. V. Nickles, and K. A. Janulewicz, "Demonstration of a 2-ps transient x-ray laser," Phys. Rev. A 65, 033810/1-4 (2002).

9. R. D. Guenther, "Parametric characterization of a light guide," in Modern Optics (Wiley, New York, 1990), pp. 163171.

10. L. B. Da Silva, T. W. Barbee, Jr., R. Cauble, P. Celliers, D. Ciarlo, S. Libby, R. A. London, D. Matthews, S. Mrowka, J. C. Moreno, D. Ress, J. E. Trebes, A. S. Wan, and F. Weber, Phys. Rev. Lett. 74, 3991-3994 (1995).

11. P. Celliers, F. Weber, L. B. Da Silva, T. W. Barbee, Jr., R.
Cauble, A. S. Wan, and J. C. Moreno, Opt. Lett. 20, 19071909 (1995).

12. V. N. Shlyaptsev, P. V. Nickles, T. Schlegel, M. P. Kalashnikov, and A. L. Osterheld, "Table-top x-ray laser pumped with subnanosecond and picosecond pulses," in Ultrashort Wavelength Lasers II, S. Suckewer, ed., Proc. SPIE 2012 , 111-118 (1994).

13. C. D. Decker, R. A. London, J. A. Harte, L. V. Powers, and J. E. Trebes, "Electron-density measurements in hohlraums using soft-x-ray deflectometry,” Phys. Rev. E 57, 5952-5957 (1998). 